\title{
Effects of Gold Nanorods on Nonlinear Properties of Graphene Films Using Z-Scan Technique
}

\author{
Thekrayat Al Abdulaal $^{1}$, Morgan Ware ${ }^{2}$, Mourad Benamara ${ }^{1}$, Alex Biris ${ }^{3}$, Viney Saini ${ }^{3}$ and Gregory Salamo ${ }^{1}$ \\ 1. Department of Physics, Institute for Nanoscience and Engineering, University of Arkansas, Fayetteville AR 72701, USA \\ 2. Department of Electrical Engineering, University of Arkansas, Fayetteville AR 72701, USA \\ 3. Center of Integrative Nanotechnology Sciences, University of Arkansas at Little Rock, Little Rock AR 72204, USA
}

\begin{abstract}
Two dimensional nanomaterials, specifically graphene, can play a significant role in various photonic and electronic devices. This is especially true in handling the enormous heat in high density electronics and in nonlinear optics when using high power lasers. To model these systems it is important to know the thermal-optical properties of graphene. In this paper, we report on the thermal and optical linear and nonlinear properties of graphene materials using Z-scan system. In particular, we explore the thermo-optical properties of graphene, with and without gold nanorods (AuNRs). The obtained results illustrate that the addition of gold nanorods causes a significant change in thermal nonlinear refractive index coefficients of graphene, due to the plasmonic enhancements.
\end{abstract}

Key words: Graphene, gold nanorods, nonlinear properties, Z-scan.

\section{Nomenclature}

$\begin{array}{ll}\mathrm{n}_{1}, \mathrm{n}_{2}: & \text { Nonlinear refractive index } \\ \beta: & \text { Nonlinear absorption coefficient } \\ \mathrm{dn} / \mathrm{dT}: & \text { Thermo-optical coefficient } \\ \mathrm{cw}: & \text { Continuous wave } \\ \text { AuNRs: } & \text { Gold nanorods } \\ \text { NFG: } & \text { Nonfunctionalized graphene } \\ \text { AuNFG: } & \begin{array}{l}\text { Nonfunctionalized graphene with gold } \\ \text { nanorods }\end{array}\end{array}$

\section{Subscripts}

$\begin{array}{ll}\mathrm{T}_{\mathrm{N}}: & \text { Normalized transmission } \\ \mathrm{nm}: & \text { Nanometer } \\ \Delta \mathrm{T}: & \text { Temperature gradient } \\ \Delta \mathrm{n}: & \text { Refraction change }\end{array}$

\section{Introduction}

The rapid improvement in high power electronics and high power lasers while simultaneously becoming smaller and smaller has led to serious heat issues. As a result, it is extremely hard to get higher performance unless this heat is efficiently removed. One possibility

Corresponding author: Thekrayat Al Abdulaal, Ph.D., research fields: graphene, nonlinear optics physics. to help reduce hot spots is to use graphene as a heat spreading material. In addition, due to its very high thermal conductivity, the growing accessibility of two dimensional nanostructured materials, especially graphene, with precise control on the thermal and optical properties has produced extensive attention in various applications, including biomolecular and chemical sensing, thermal storage solar cells, photonics devices, cooling systems, and photo-thermal therapy [1].

Graphene is a 2D material made of a few layers of carbon atoms that are arranged in the shape of a hexagon [2]. The strength of graphene is due to the $\mathrm{sp}^{2}$ bonds among the carbon atoms, as illustrated in Fig.1. To specify, $0.14 \mathrm{~nm}$ is the distance between carbon atoms, and $0.34 \mathrm{~nm}$ is the distance between graphene sheets. For these reasons and because of the thermally induced nonlinear index change that can be produced, research on the thermal and optical nonlinear properties of graphene is very important.

Our research on graphene has demonstrated that, nanoparticles can enhance the nonlinear thermal and 


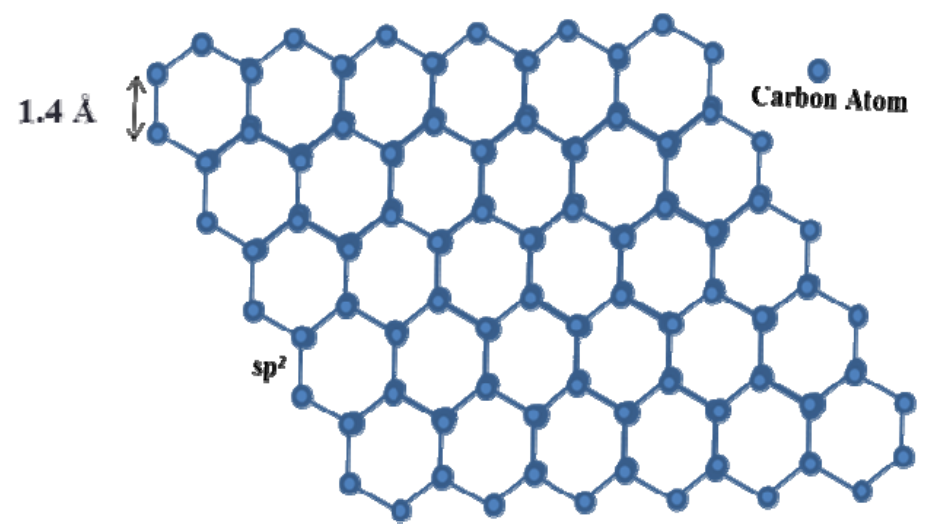

Fig. 1 Hexagonal structure of 2D graphene material.

optical properties. The third order change in the nonlinear refractive index of the material can be very high due to the temperature change. Here the refractive index $(n)$ could be expressed as $n(T)=n_{0}+n_{1} T+n_{2}$ $\mathrm{T}^{2}$; where $\mathrm{n}_{0}$ is the material refraction, $\mathrm{n}_{1}$ and $\mathrm{n}_{2}$ are the thermal linear and nonlinear term of refractive index [1]. Moreover, the thermal nonlinear properties can play an important role for continuous wave (cw) lasers due to optical absorption and corresponding thermal lensing effects. The main goal of this research was to investigate the thermal nonlinearities of non-functionalized graphene thin films without and with gold nanorods (NFG and AuNFG) on glass substrates, including nonlinear absorption coefficient $(\beta)$, thermal nonlinear refractive index coefficients $\left(n_{1}\right.$ and $\left.\mathrm{n}_{2}\right)$, and thermo-optical coefficient (dn/dT). With this goal in mind, the objective was to first build a precise, simple, and sensitive technique, Z-scan system, to investigate and understand the thermal nonlinear properties, which are dependent on the laser power and temperature.

\section{Experimental Setup}

In the nonlinear regime, Z-scan is an optical technique that is mainly utilized for characterizing the properties of the third-order nonlinear optical materials. Z-scan technique is considered a standard method due to its simple layout, high sensitivity, and easiness to analyze data. The basic principle of the Z-scan method is measuring the transmittance of $\mathrm{TEM}_{00}$ Gaussian laser light in the far field as a function of the sample position $(\mathrm{z})$ in relation with the focal point [1], as illustrated in Fig. 2. Here in the Z-scan system, a continuous wave (cw) of an argon ion $\left(\mathrm{Ar}^{+}\right)$laser beam was used as the excitation source at wavelength of $514 \mathrm{~nm}$ in resonance with gold nanorods.

The sample was fixed on a micrometer translation stage and moved in the propagation direction $(\mathrm{z})$ of a Gaussian beam that was narrowly focused. After that, the transmitted signal, passing via the materials as a function of its position was observed using a photo detector placed behind a small aperture in the far field. There are two parts of the Z-scan method: one with open aperture is sensitive to measure nonlinear absorption where the whole beam is detected, and the other is with closed aperture that is sensitive to measure thermal nonlinear refraction and thermo-optical coefficient, where the aperture and the far field detector make it possible to detect the small beam distortion, any expansion or shrinking in the original laser beam [3].

\section{Experimental Results}

\subsection{Linear Measurements}

UV-Visible spectrophotometer was used to linearly optically characterize all graphene films as well as goldnanorods in the spectral range from $200 \mathrm{~nm}$ to 


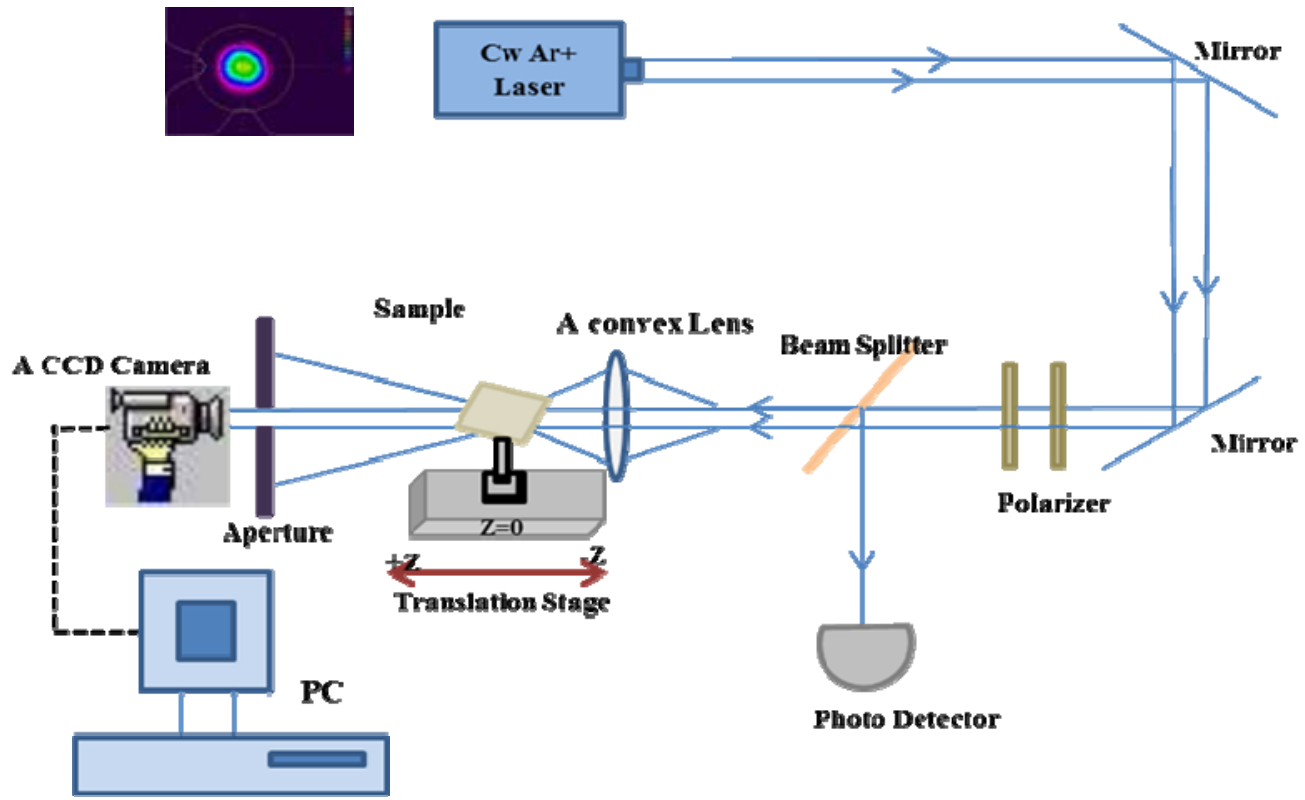

Fig. 2 Illustration of experimental setup of the Z-scan system.

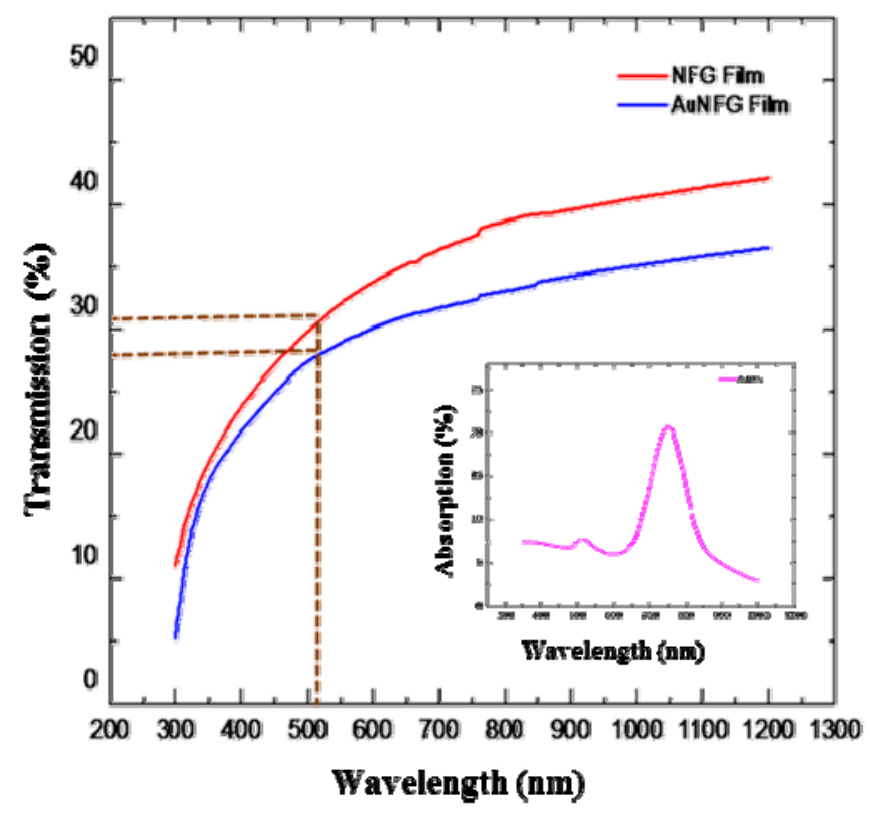

Fig. 3 Transmittance spectra of NFG and AuNFG films, insert is the absorption spectrum of gold nanorods.

1,200 $\mathrm{nm}$ at room temperature. Fig. 3 shows that the transmittance of nonfunctionalized graphene with gold nanorods is getting smaller compared to the film without gold, also the transverse plasmonic resonance location of gold nanorods was indicated to be around $500 \mathrm{~nm}$. This concludes that surface plasmonic absorption of gold nanorods enhances the light absorption more in the AuNFG film.
Moreover, AFM (atomic force microscopy) was used to determine the average thickness of graphene films and to initially confirm the presence of gold nanorods in the AuNFG film. The height and roughness of all graphene thin film samples on glass substrates were defined by AFM imaging and profiling at ambient condition, where the color scale indicates the height variation as illustrated in Fig. 4(a) for 


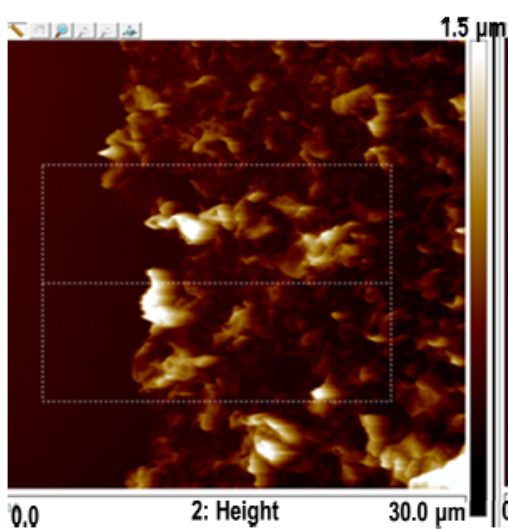

(a) NFG

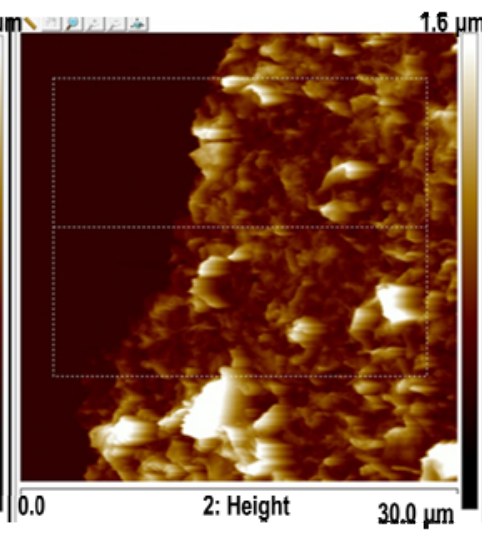

(b) AuNFG

Fig. 4 AFM images acquired for NFG in (a) and AuNFG in (b) films.

NFG and Fig. 4(b) for AuNFG. To clarify, the AFM image of the AuNFG thin film sample shows that gold nanorods partially covered the NFG film. The AuNRs appear more likely as bright dots at higher regions.

The gold nanorods were also examined by TEM (transmission electron microscopy) in order to accurately determine their size and shape. Fig. 5 shows the TEM micrograph of the gold nanorods and the average length was $36 \pm 3 \mathrm{~nm}$, and the average diameter was $12 \pm 2 \mathrm{~nm}$, with an AR (aspect ratio) of around 3. The TEM image demonstrated a high quality of uniformly grown gold nanorods.

These values of AuNR sizes were taken into consideration when estimating the concentration of gold nanorods in graphene thin film samples, assuming a 2D sheet of AuNRs did not affect the average thickness. ESEM (environmental scanning electron microscopy) was used to estimate the AuNRs density. As shown in Fig. 6, the bright contrast locations of the SEM image indicated the presence of graphene layers together with gold nanorods. It can be seen that the graphene surface was decorated by AuNRs, partially covering approximately $11 \%$ of the film. A statistical analysis showed that the number of gold nanorods per unit area of the film was determined to be around $8.6 \times 10^{11} \mathrm{NRs} / \mathrm{cm}^{2}$. All linear measurements confirm that the Z-scan system with $514 \mathrm{~nm}$ wavelength was close to the AuNRs plasmonic resonance.

\subsection{Nonlinear Absorption Measurements}

Data taken using apparatus in Fig. 2 can be made with the aperture to measure nonlinear refraction and without the aperture to measure nonlinear absorption. In the case of open aperture Z-scan, the nonlinear absorption $(\beta)$ at various laser input power-158, 303, 525, 620, and $767 \mathrm{~mW}$, was studied for the AuNFG film, as shown in Fig. 7. The experimental data points were shown together with the theoretical fitting curve in the solid line. The theoretical equation to fit the normalized transmittance in the far field is given by [4]:

$$
\mathrm{T}_{\mathrm{N}}(\mathrm{z}, \mathrm{t})=-\mathrm{q}_{0}\left[2.83\left(1+\left(\mathrm{z} / \mathrm{z}_{0}\right)^{2}\right)\right]^{-1}+1
$$

Here $\mathrm{z}$ is the material position, $\mathrm{z}_{0}$ is the Rayleigh length, and the complex parameter $\left(\mathrm{q}_{0}\right)$ is linearly related to the nonlinear absorption $(\beta)$ by:

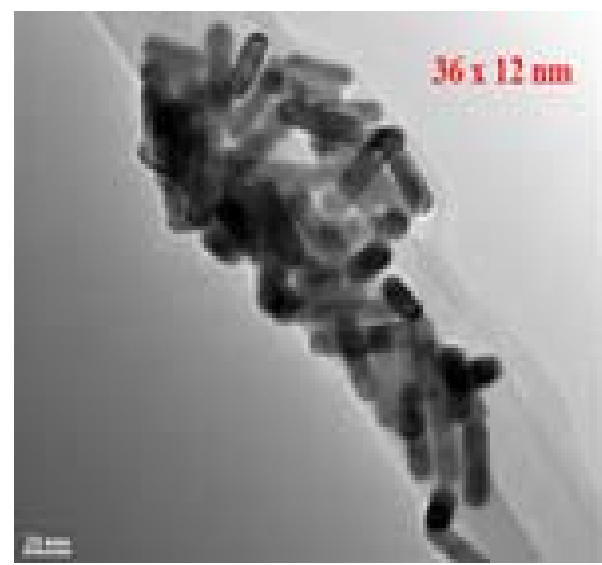

Fig. 5 TEM image of AuNRs on a carbon grid, and the scale bar indicates $20 \mathrm{~nm}$. 

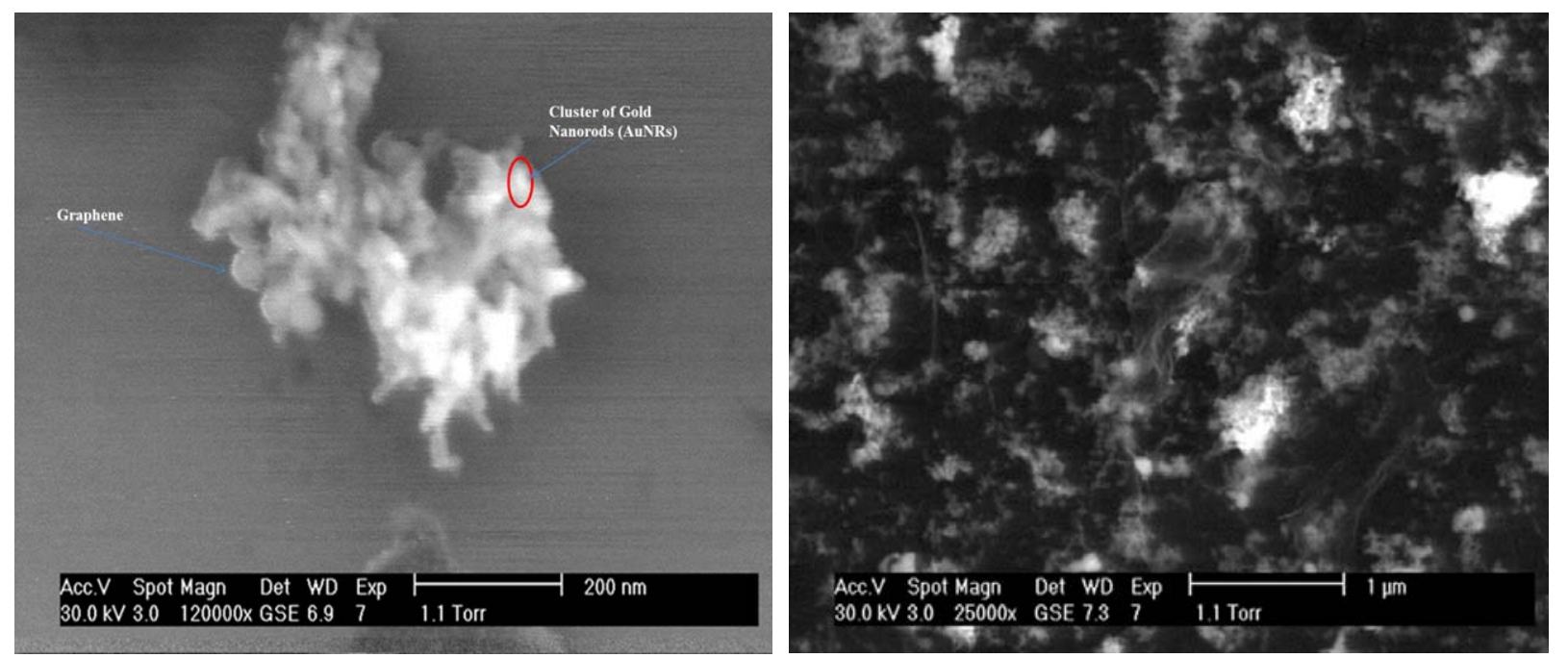

Fig. 6 ESEM images of the AuNFG Film.

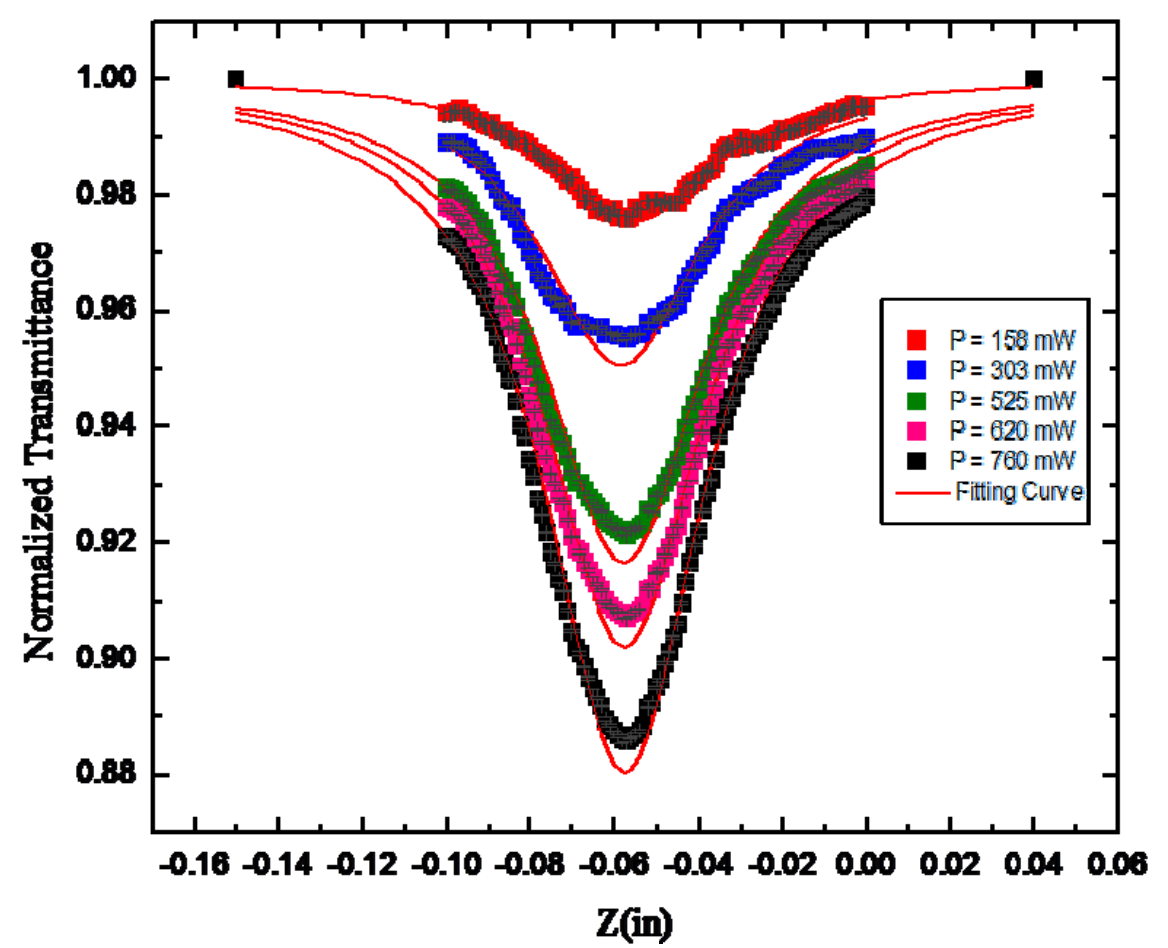

Fig. 7 Illustration of experimental data of open aperture Z-Scan measurements for the AuNFG film at various applied laser power.

$$
\mathrm{q}_{0}=\beta \mathrm{I}_{0} \mathrm{~L}_{\mathrm{eff}}\left(\left|\mathrm{q}_{0}\right|<<1\right)
$$

Here $\mathrm{I}_{0}$ is the laser intensity at the focus, and $\mathrm{L}_{\text {eff }}$ is the effective length of the sample.

$$
\mathrm{L}_{\text {eff }}=\left(1-\mathrm{e}^{-\alpha \mathrm{L}}\right) / \alpha
$$

Here, $L$ and $\alpha$ are the martial thickness and linear absorption coefficient, respectively.

The experimental results agreed with the theoretical model in which there is a smooth symmetric valley around the lens focus and as the applied input laser power increases more absorption occurs inside the material, indicating a larger valley. It can also be seen here that the nonlinear absorption $(\beta)$ of the graphene sample with gold is higher compared to that value without AuNRs, as illustrated in Fig. 8. 


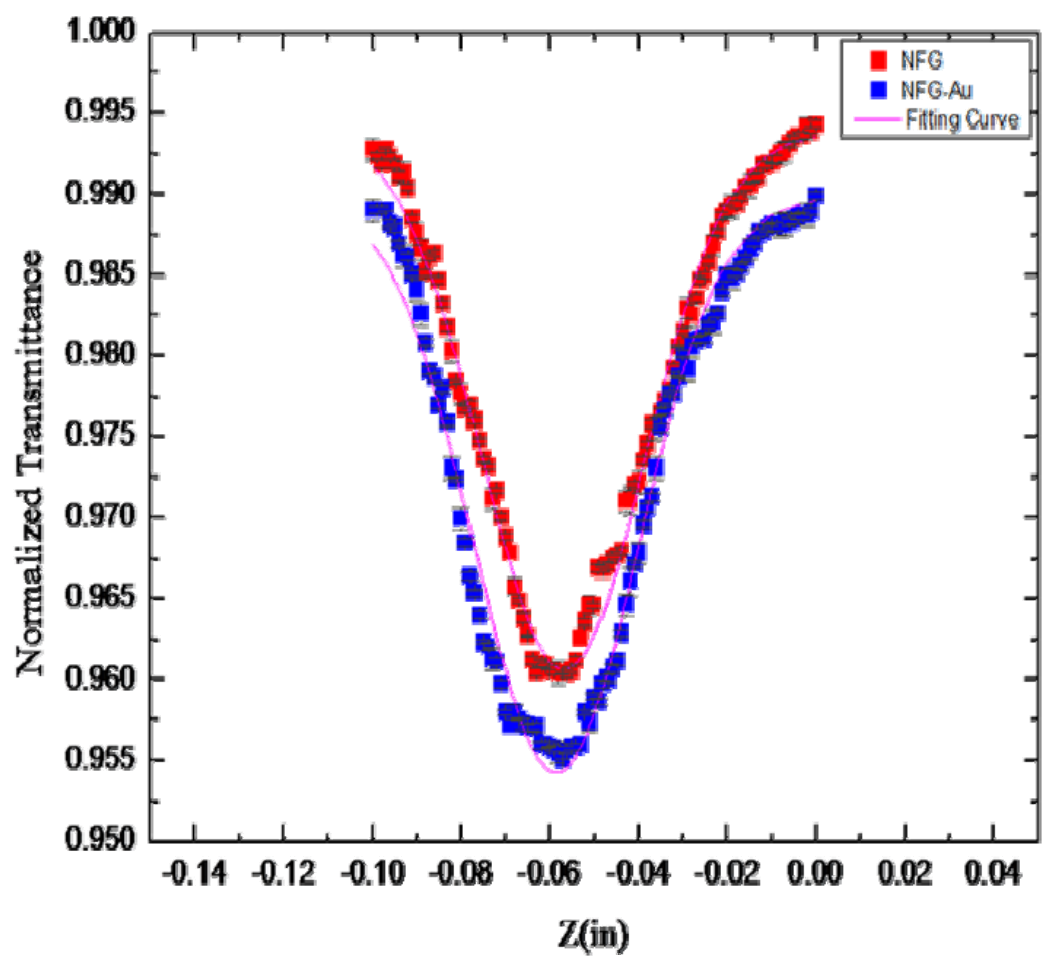

Fig. 8 Open aperture results for the NFG and Au-NFG samples at a $303 \mathrm{~mW}$ power of a cw $\mathrm{Ar}^{+}$laser.

The value of $\beta$ was calculated based on the slope of the linear curve in equation taking in account that:

$$
\alpha=\alpha+\beta \mathrm{I}_{0}
$$

Thus, the $\beta$ value for NFG is calculated to be $1.58 \times$ $10^{-2}$ and to be $2.602 \times 10^{-2} \mathrm{~cm} / \mathrm{W}$ when deposited with AuNRs. For the same density of gold nanorods, the $\beta$ value of AuNRs is negligible compared to the nonlinear absorption of graphene with and without AuNRs. The results confirm that the presence of gold nanorods on the graphene sample enhances the nonlinear absorption by $50 \%$.

\subsection{Nonlinear Refraction Measurements}

In the other side, a $500 \mu \mathrm{m}$ pinhole was located before the detector to run closed aperture Z-scan and to extract the magnitude and the sign of the thermal nonlinear refractive index. Fig. 9 clarifies the closed aperture results for the AuNFG sample at various cw $\mathrm{Ar}^{+}$laser powers at $514 \mathrm{~nm}$ wavelength. All experimental data illustrate a peak followed by a valley which suggests a self- defocusing behavior, negative phase shift, directed to a negative nonlinear refraction.
Moreover, gold nanorods clearly enhance the nonlinear refraction of graphene materials as seen in Fig. 10.

The thermal lens model was excellently fit with the experimental data and was used to extract the phase shift at different laser powers. The theoretical equation to determine the change in the nonlinear refraction $\left(\mathrm{n}_{1}\right.$ and $\left.\mathrm{n}_{2}\right)$ and the thermo-optical coefficient $(\mathrm{dn} / \mathrm{dT})$ from the experimental data of Z-scan is expressed as [5]:

$$
\mathrm{NT}(\mathrm{z}, \mathrm{t})=\left[1+\left(\theta 2 \mathrm{x} /\left(1+\mathrm{x}^{2}\right)\right)\right]^{-1}
$$

where, $x=z / z_{0}$ is the normalized position, $\mathrm{dn} / \mathrm{dT}$ is related to phase change $(\theta)$ by this relation:

$$
\theta=\alpha \mathrm{PL} / \lambda \kappa[\mathrm{dn} / \mathrm{dT}]
$$

Eq. (5) defines the change in nonlinear refractive index $(\Delta \mathrm{n})$ as:

$$
\begin{gathered}
\Delta \mathrm{n}=[\mathrm{dn} / \mathrm{dT}] \Delta \mathrm{T} \\
\Delta \mathrm{n}=\mathrm{n}_{1} \Delta \mathrm{T}+\mathrm{n}_{2}(\Delta \mathrm{T})^{2}
\end{gathered}
$$

Where the temperature change $(\Delta \mathrm{T})$ is related to the laser intensity by the following equation:

$$
\Delta \mathrm{T}=\alpha \mathrm{I}_{0} \mathrm{~W}_{0}{ }^{2} / 4 \kappa
$$

Here $\mathrm{w}_{0}$ and $\kappa$ respectively are the beam waist size and the heat conductivity, $\mathrm{P}$ is the laser input power, $\mathrm{I}_{0}$ 


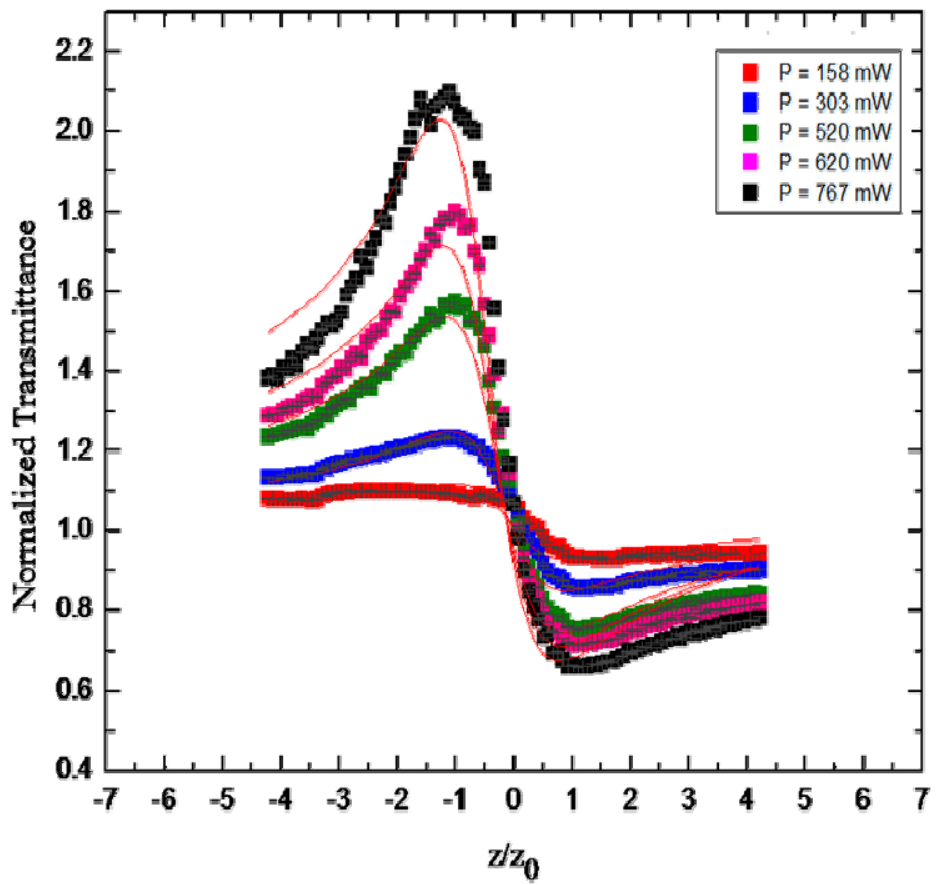

Fig. 9 Closed aperture Z-scan results data (normalized transmittance as a function of the AuFG position) at different applied laser powers; the red curves represent the theoretical fitting model.

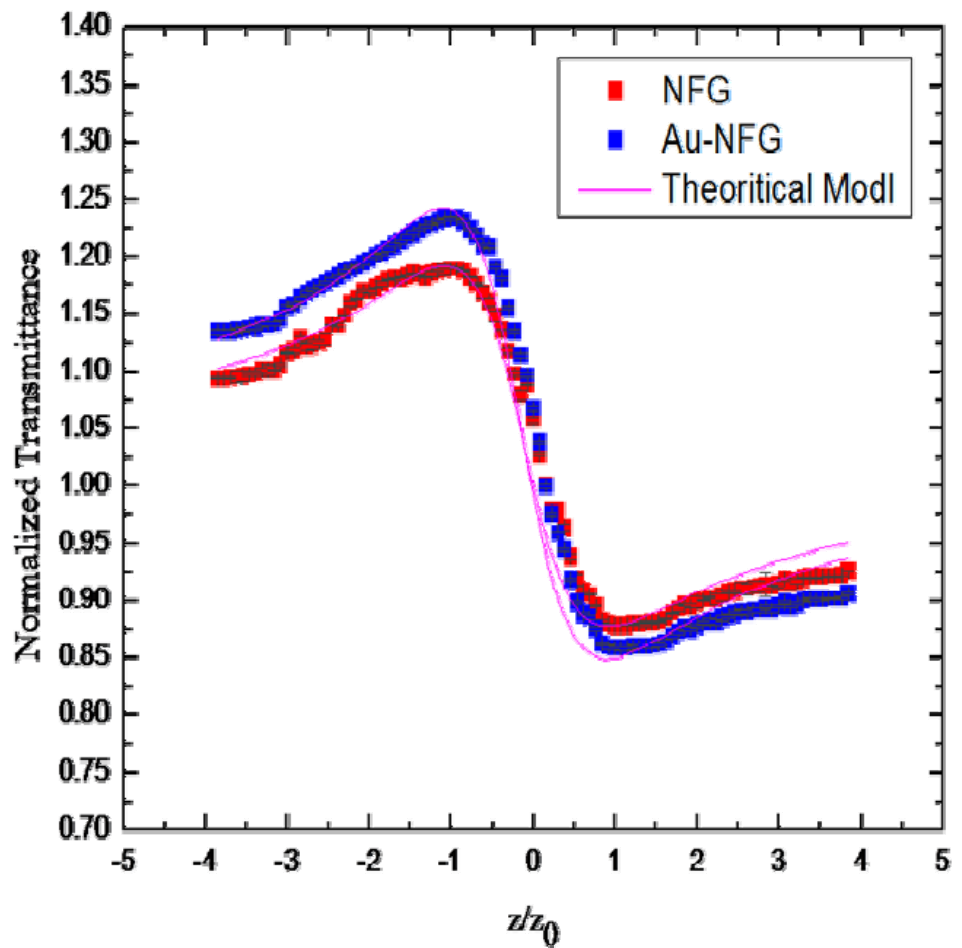

Fig. 10 Experimental data of closed aperture Z-scan for NFG and AuNFG films at $303 \mathrm{~mW}$ laser power. 
Table 1 Summary of the linear and nonlinear properties of NFG, AuNFG, and AuNRs samples at 514 nm wavelength [6].

\begin{tabular}{llll}
\hline Parameters & NFG & Au-NFG & AuNRs \\
\hline Linear absorption $(\alpha) \mathrm{cm}^{-1}$ & $2.27 \mathrm{E}+4$ & $2.97 \mathrm{E}+4$ & $0.126 \mathrm{E}+3$ \\
Linear refractive index $\left(\mathrm{n}_{0}\right)$ & 2.141 & 1.622 & 2.314 \\
Affective thickness $\left(\mathrm{L}_{\mathrm{eff}}\right) \mathrm{nm}$ & 254 & 261 & $36 \times 12$ \\
Thermal conductivity $(\mathrm{k}) \mathrm{K}[4]$ & 1,495 & 1,240 & 320 \\
Thermo-optic coefficient $(\mathrm{dn} / \mathrm{dT}) \mathrm{K}$ & $2.91 \times 10^{-4}$ & $1.387 \times 10^{-4}$ & $0.2301 \times 10^{-5}$ \\
& $\pm 2.63 \mathrm{E}-5$ & $\pm 3.72 \mathrm{E}-5$ & $\pm 8.33 \mathrm{E}-7$ \\
Nonlinear refractive index $\left(\mathrm{n}_{1}{ }^{\prime}\right) \mathrm{cm}^{2} / \mathrm{W}$ & $-1.5224 \times 10^{-6}$ & $-6.713 \times 10^{-6}$ & $-0.1 \times 10^{-9}$ \\
& $\pm 2.37 \mathrm{E}-8$ & $\pm 3.12 \mathrm{E}-7$ & $\pm 1.65 \mathrm{E}-11$ \\
Nonlinear refractive index $\left(\mathrm{n}_{2}{ }^{\prime}\right) \mathrm{cm}^{4} / \mathrm{W}^{2}$ & $-0.243 \times 10^{-12}$ & $-1.915 \times 10^{-12}$ & $-0.042 \times 10^{-16}$ \\
& $\pm 0.0038 \mathrm{E}-12$ & $\pm 0.00643 \mathrm{E}-12$ & $\pm 0.023 \mathrm{E}-16$ \\
Nonlinear absorption coefficient $(\beta)(\mathrm{cm} / \mathrm{W})$ & $1.58 \times 10^{-2}$ & $2.602 \times 10^{-2}$ & $2.15 \times 10^{-5}$ \\
& $\pm 1.67 \mathrm{E}-3$ & $\pm 2.76 \mathrm{E}-3$ & $\pm 3.19 \mathrm{E}-6$ \\
Changing in refractive index $(\Delta \mathrm{n})$ & 0.0897 & 0.0312 & $0.478 \times 10^{-3}$ \\
\hline
\end{tabular}

is the laser intensity at the focus, $\lambda$ is the laser wavelength, $\mathrm{L}$ is the material thickness and $\alpha$ is the material linear absorption coefficient.

The fitting curve has allowed pulling out the nonlinear values of NFG coefficients to be $\mathrm{dn} / \mathrm{dT}=$ $-2.09 \mathrm{E}-4 / \mathrm{K}, \Delta \mathrm{n}=0.0897, \mathrm{n}_{1}{ }^{\prime}$ to be $-1.52 \times 10^{-6} \pm 2.37$ $\mathrm{E}-8 \mathrm{~cm}^{2} / \mathrm{W}$ and $\mathrm{n}_{2}{ }^{\prime}=-0.243 \times 10^{-12} \pm 0.0038 \mathrm{E}-12$ $\mathrm{cm}^{4} / \mathrm{W}^{2}$. Also, the thermo-optical coefficient of the AuNFG film were found to be of the order -1.387 E-4/K and the value of the refraction change $(\Delta \mathrm{n})$ was estimated to be 0.00312 . From the nonlinear fitting curve for the closed aperture Z-scan data the nonlinear refraction (n1 ' and $\mathrm{n} 2^{\prime}$ ) values were determined, with a $9.74 \mu \mathrm{m}$ waist size of the Ar+ laser beam at the lens focus, to be $-6.713 \times 10^{-6} \pm 3.12 \mathrm{E}-7 \mathrm{~cm}^{2} / \mathrm{W}$ and $-1.92 \times$ $10^{-12} \pm 0.00643 \mathrm{E}-12 \mathrm{~cm}^{4} / \mathrm{W}^{2}$, respectively. It was observed that the gold nanorods increased the thermal nonlinear refraction of the AuFG film by a factor of five, compared to that value for the NFG sample, and much larger (4 orders of magnitude) than that for AuNRs. Also, there was enormous enhancement in the thermo-optic coefficient of AuNFG film owing to the presence of AuNRs in the graphene material.

Thus, the improvement of the thermal nonlinearities for AuNFG is apparently due to the gold nanorods producing localized heating around the graphene material. The nonlinear refraction and absorption were successfully evaluated for the studied samples (NFG,
AuNRs, and AuNFG) using 514 nm laser wavelength, as shown in Table 1.

\section{Conclusions and Future Work}

This research reports the first measurement on the nonlinear thermal and optical properties of graphene with and without gold nanorods in the transverse surface plasmon absorption region (514 $\mathrm{nm}$ wavelength), by using the Z-scan technique. It has been observed that the nonlinear absorption and nonlinear index of gold nanorods embedded in graphene differ remarkably with those of graphene alone. This study concludes that the investigated graphene flakes and $\mathrm{Au}$ nanorods have nonlinear effects and that the AuNFG film presents a large or enhanced thermal nonlinear refractive index. This value was about five times larger than the nonlinear refraction of the graphene without gold and much larger (4 order of magnitude) than that for $\mathrm{Au}$ nanorods alone. There was also an enormous enhancement on the thermo-optic coefficient of the composite film owing to the presence of $\mathrm{Au}$ in the graphene material.

The conclusion is that the ability of graphene to be used as an excellent heat spreader increases as the temperature increases because the thermal nonlinearity of graphene increases linearly with the applied laser powers. Also, investigation on the nonlinear optical properties of graphene must take into 
account a significant nonlinear refractive index due to the thermal effects (heat). In terms of impact, the structure is a promising candidate for photonic applications, such as optical data storage, optical limiting, information processing, and optical communication $[1,7]$. Future studies might pursue the nonlinear optical properties of the same materials using high power pulsed laser excitation at different laser wavelengths. In addition, the effect of gold nanorods in enhancing the graphene nonlinearities could be investigated in and out of resonance.

\section{Acknowledgments}

The authors would like to thank Saudi Arabia Cultural Mission (SACM) and physics department at University of Arkansas for their financial support. A lot of thanks go to Tim Morgan, Mohamed Zamani, and Xian $\mathrm{Hu}$ for their help and significant discussions. Thanks to all faculty, students and employees at the University of Arkansas, especially in the Institute for Nano Science and Engineering and in the sponsored program office.

\section{References}

[1] Sheik-Bahae, M., Said, A. A., and van Stryland, E. W. 1990. "Sensitive Measurement of Optical Nonlinearities Using a Single Beam." IEEE J .Quan. Electron. 26: 760-9.

[2] Zhu, Y., Murali, S., Cai, W., Li, X., Suk, J. W., Potts, J. R., and Ruoff, R. S. 2010. "Graphene and Graphene Oxide: Synthesis, Properties, and Applications." Adv. Mater. 22 (35): 3906-24.

[3] Badran, H., and Al-Fregi., A. 2012. "Synthesis and Study of Nonlinear Optical Properties of a New Azo Dye by Z-scan Technique." International Journal of Physics and Research 2 (2): 15-25.

[4] Falconieri, M. 1999. "Thermo-optical Effects in Z-scan Measurements Using High-Repetition-Rate Lasers." Journal of Optics A: Pure and Applied Optics 1 (6): 662.

[5] Wang, J., Zhu, L., Chen, J., Li, B., and Thong, J. T. 2013. "Suppressing Thermal Conductivity of Suspended Tri-layer Graphene by Gold Deposition." Advanced Materials 25 (47): 6884-8.

[6] Al Abdulaal, T. 2016. "Measuring Nonlinear Properties of Graphene Thin Films Using Z-Scan Technique." University of Arkansas.

[7] Nima, Z. A., Lahiani, M. H., Watanabe, F., Xu, Y., Khodakovskaya, M. V., and Biris, A. S. 2014. "Plasmonically Active Nanorods for Delivery of Bio-active Agents and High-sensitivity SERS Detection in Planta." RSC Adv. 4 (110): 64985-93. 